\title{
Phototermal Spectra of Inhomogeneous Coatings
}

\author{
M. Popovic, S. Galovic*, And Z. Stojanovic \\ "Vinca" Institute of Nuclear Sciences, Belgrade, Serbia
}

In this paper we demonstrate a theoretical study for photothermal measurements on inhomogeneous coatings. First, a general photothermal mathematical model for thermal gradient materials is presented. Then, we discuss the effects of inhomogeneous thermal properties in photothermal amplitude and phase spectra of coatings. Finally, a method for quantitative depth profiling that makes use of prior knowledge about the type of profile existing in a sample to reduce the instabilities associated with the mathematically ill conditioned task is demonstrated.

PACS numbers: 42.62.Eh, 44.05.+e, 44.10.+i, 68.60.Dv

\section{Introduction}

Photothermal (PT) measurement techniques are being intensively developed and applied with increased success in the measurement of thermal, optical and other related physical properties, as well as for the investigation of subsurface structure and macroscopic defects of various samples.

Photothermal methods are based on direct or indirect measurements of temperature variations, which occur because of generation and transfer of the heat produced by excitation of a testing structure by an external source of energy. Hence, a correct description of thermal transport processes is necessary for the analysis of the measured signals and for their application in the determination of the physical sample properties. All the existing models of photothermal signals are based on the solutions of the classical heat conduction equation that neglects the thermal memory effects. However, a large group of versatile materials with significant thermal memory (such as complex biological materials, polymers, metals excited by very short laser pulses, dielectrics and semiconductors in up-to-date electronic devices, etc.) are extensively used in science and technology. The heat transfer within these media and, implicitly, photothermal effects, cannot be described by the classical law of heat conduction [1].

In the last few years, much effort has been made in the study of photothermal effects in thermally inhomogeneous solids [2-5]. The main problem arises from the impossibility of solving the inhomogeneous heat conduction equation in a general case. Unfortunately, a strict mathematical interpretation of thermal wave propagation in solids is essential if one wishes to give a good theoretical description of any photothermal phenomenon. There are some results for classical thermal heat conduction equation, especially for discrete inhomogeneous materials $[6,7]$, but there is no result discussing the influence of thermal memory.

In order to achieve this goal several attempts have been made. The common approach was to find approx-

* corresponding author; e-mail: bobagal@vinca.rs imate solutions of strictly formulated heat conduction problems. Various numerical procedures were reported and, recently, an effective medium theory was also applied. However, there are still several disadvantages that limit the practical use of these methods. First, approximation conditions must be carefully examined in each calculation concerning a material with different thermal properties or geometry. In addition, results of numerical simulations do not provide solutions in a form of analytical expressions from which a comprehensive analysis of photothermal effects could be performed.

On the other hand, the description of heat conduction in inhomogeneous solids can be evaluated using exact analytical solutions of the heat conduction equation, but for the approximately formulated starting photothermal problem. This is, in fact, an inverted approach with respect to the methods we have already mentioned where approximations were made during calculation.

In this paper we present a general photothermal mathematical model for thermal gradient materials [8]. The case of constant thermal diffusivity and linear variable heat capacity on inhomogeneous coatings is discussed, which is good approximation of thermal inhomogeneous thin layers [9]. Finally, a method for quantitative depth profiling that makes use of prior knowledge about the type of profile existing in a sample to reduce the instabilities associated with the mathematically ill conditioned task is demonstrated [10].

\section{Theory}

Thermal waves are excited in a two-layer system, consisting of a thin coating on a substrate, by the absorption of an intensity-modulated laser beam, $S(t)=S_{0} f(t)$. Assuming opaque coating surface at the wavelength of the laser beam, the absorbed part of the incident laser beam intensity is transformed into the heat just at the surface, so the heat source is given by the expression $S(t)=S_{0} f(t) \delta(x)$.

The common configuration for most photothermal experiments is schematically represented in Fig. 1. The investigated sample (coating), which has an arbitrary heat capacity $c(x)$ and constant thermal diffusivity $D_{\mathrm{T} i}$, is 
surrounded by two media, usually gas $(g)$ and backing (b) (substrate). Assuming a large heating spot diameter, the one-dimensional temperature distribution in the coating is possible.

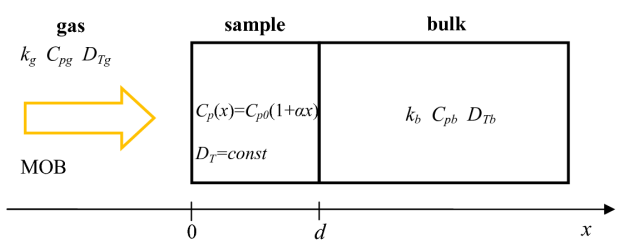

Fig. 1. Schematic representation of the most common configuration in photothermal experiments.

The heat conduction process is then fully described by a system of partial differential equations (PDEs), for each medium, together with boundary conditions describing continuity of heat flux $q(x, t)$ and temperature $\vartheta(x, t)$ at the interfaces between media. For the thermally homogeneous bounding media with no heat sources or sinks conduction of heat is described by hyperbolic PDEs with constant coefficients

$$
\begin{aligned}
& \frac{\partial^{2} \vartheta(x, t)}{\partial x^{2}}-\frac{1}{D_{\mathrm{T} i}}\left[\frac{\partial \vartheta(x, t)}{\partial t}+\tau_{i} \frac{\partial^{2} \vartheta(x, t)}{\partial t^{2}}\right]=0, \\
& i=g, b, \\
& q(x, t)+\tau_{i} \frac{\partial q(x, t)}{\partial t}=-k_{i} \frac{\partial \vartheta(x, t)}{\partial x}, \quad i=g, b .
\end{aligned}
$$

$\tau_{i}$ is thermal relaxation time which describes thermal memory. For the arbitrary inhomogeneous sample we have inhomogeneous PDEs

$$
\begin{aligned}
& \frac{\partial}{\partial x}\left[C_{p}(x) \frac{\partial \vartheta(x, t)}{\partial x}\right]-\frac{C_{p}(x)}{D_{\mathrm{T}}}\left[\frac{\partial \vartheta(x, t)}{\partial t}+\tau \frac{\partial^{2} \vartheta(x, t)}{\partial t^{2}}\right] \\
& =-\left[S(x, t)+\tau \frac{\partial S(x, t)}{\partial t}\right], \\
& q(x, t)+\tau \frac{\partial q(x, t)}{\partial t}=-D_{\mathrm{T}} C_{p}(x) \frac{\partial \vartheta(x, t)}{\partial x} .
\end{aligned}
$$

The application of the Laplace transformation method, assuming initial conditions of zero temperature, reduces the starting problem (Eqs. (1)-(4)) to a system of ordinary differential equations (ODEs):

$$
\begin{aligned}
& \frac{\mathrm{d}^{2} \tilde{\theta}(x)}{\mathrm{d} x^{2}}-\sigma_{i}^{2}=0, \quad i=g, b, \\
& \tilde{Q}(x)=-\frac{k_{i}}{1+\tau p} \frac{\mathrm{d} \tilde{\theta}(x)}{\mathrm{d} x}, \quad i=g, b . \\
& y^{2} \frac{\mathrm{d}^{2} \tilde{\theta}(y)}{\mathrm{d} y^{2}}+y \frac{\mathrm{d} \tilde{\theta}(y)}{\mathrm{d} y}-\frac{y^{2} \tilde{\sigma}^{2}}{\alpha^{2}} \tilde{\theta}(y) \\
& =-\frac{y}{C_{p 0} \alpha^{2}} \tilde{S}(1+\tau p), \\
& Q(x)=-\frac{D_{\mathrm{T}}}{1+\tau p} C_{p}(x) \frac{\mathrm{d} \tilde{\theta}(x)}{\mathrm{d} x} .
\end{aligned}
$$

with homogeneous boundary conditions and conditions of continuity on interfaces. Tilda sign denotes the complex values (Laplace transforms). Equation (7) is second order inhomogeneous Bessel's differential equation [11], that is delivered using $C_{p}(x)=C_{p 0}(1+\alpha x)$. In previous equations $\tilde{\sigma}$ is complex conductivity, $\tilde{\sigma}_{i}=\sqrt{\frac{p}{D_{\mathrm{T} i}}\left(1+\tau_{i} p\right)}$ for $i=\mathrm{g}, \mathrm{b}, \tilde{\sigma}=\sqrt{\frac{p}{D_{\mathrm{T}}}(1+\tau p)}$ for sample, and $y=1+\alpha x$.

General solution of the ODE system (Eqs. (5)-(8)) is in the following form:

$$
\begin{aligned}
& \tilde{\theta}(x)=A_{1} \mathrm{e}^{\sigma_{g} x}+A_{2} \mathrm{e}^{-\sigma_{g} x}, \quad x<0, \\
& \tilde{\theta}(x)=A_{3} J_{0}(\xi)+A_{4} Y_{0}(\xi), \quad 0 \leq x<d, \\
& \tilde{\theta}(x)=A_{5} \mathrm{e}^{\sigma_{b} x}+A_{6} \mathrm{e}^{-\sigma_{b} x}, \quad x \geq d .
\end{aligned}
$$

$A_{i}$ are constants of integration which should be determined from boundary conditions and conditions of continuity on interfaces, $J_{0}$ and $Y_{0}$ are Bessel's functions of complex argument $\xi$, zeros order, first and second type respectively, and $\xi=j \frac{\sigma}{\alpha} y$.

The resulting equations for the complex temperature distribution in a thermally inhomogeneous sample are

$$
\begin{aligned}
& \tilde{\theta}(x)=\frac{S_{0} \tilde{F}(1+\tau p)}{\tilde{\sigma} D_{\mathrm{T}} C_{p 0}} \frac{b \pi_{1}+\mathrm{j} \pi_{2}}{g b \pi_{1}+\mathrm{j} g \pi_{2}-\mathrm{j} b \pi_{3}-\pi_{4}} \mathrm{e}^{\tilde{\sigma}_{g} x}, \quad x<0 \\
& \tilde{\theta}(x)=\frac{S_{0} \tilde{F}(1+\tau p)}{\tilde{\sigma} D_{\mathrm{T}} C_{p 0}} \frac{\left[b Y_{0}\left(\xi_{d}\right)-\mathrm{j} Y_{1}\left(\xi_{d}\right)\right] J_{0}(\xi)-\left[b J_{0}\left(\xi_{d}\right)-\mathrm{j} J_{1}\left(\xi_{d}\right)\right] Y_{0}(\xi)}{g b \pi_{1}+\mathrm{j} g \pi_{2}-\mathrm{j} b \pi_{3}-\pi_{4}}, \quad 0 \leq x<d, \\
& \tilde{\theta}(x)=\frac{S_{0} \tilde{F}(1+\tau p)}{\tilde{\sigma} D_{\mathrm{T}} C_{p 0}} \frac{\mathrm{e}^{-\tilde{\sigma}_{b}(x-d)}}{g b \pi_{1}+\mathrm{j} g \pi_{2}-\mathrm{j} b \pi_{3}-\pi_{4}}, \quad x \geq d .
\end{aligned}
$$

Here are

$$
\begin{aligned}
& \pi_{1}=J_{0}\left(\xi_{0}\right) Y_{0}\left(\xi_{d}\right)-Y_{0}\left(\xi_{0}\right) J_{0}\left(\xi_{d}\right), \\
& \pi_{2}=J_{1}\left(\xi_{d}\right) Y_{0}\left(\xi_{0}\right)-Y_{1}\left(\xi_{d}\right) J_{0}\left(\xi_{0}\right),
\end{aligned}
$$

$$
\begin{aligned}
& \pi_{3}=J_{0}\left(\xi_{d}\right) Y_{1}\left(\xi_{0}\right)-Y_{0}\left(\xi_{d}\right) J_{1}\left(\xi_{0}\right), \\
& \pi_{4}=J_{1}\left(\xi_{d}\right) Y_{1}\left(\xi_{0}\right)-Y_{1}\left(\xi_{d}\right) J_{1}\left(\xi_{0}\right),
\end{aligned}
$$

then 


$$
g=\frac{k_{g} \tilde{\sigma}_{g}}{\left(1+\tau_{g} p\right)} \frac{(1+\tau p)}{\tilde{\sigma} D_{\mathrm{T}} C_{p 0}}
$$

and

$$
b=\frac{k_{b} \tilde{\sigma}_{b}}{\left(1+\tau_{b} p\right)} \frac{(1+\tau p)}{\tilde{\sigma} D_{\mathrm{T}} C_{p 0}(1+\alpha d)} .
$$

\section{Results and discussion}

There are two interesting cases which can be extracted from the point of view of PT analyzing, and these are thin films and surface layers or coatings. Thin films can be surrounded by gas and PT signal can be obtained on the both sides of the sample $(g \approx b \approx 0)$. In the other case thermal parameters are continuously varying to some depth where they get values of bulk $(b \approx 1)$. For PT techniques of coatings, the relevant feature is temperature variation on a sample surface. It can be evaluated from Eq. (13) for $x=0$ :

$$
\tilde{R}_{p}(0)=\frac{S_{0} \tilde{F}(1+\tau p)}{\tilde{\sigma} D_{\mathrm{T}} C_{p 0}} \frac{J_{0}\left(\xi_{0}\right) Y_{0}\left(\xi_{d}\right)-Y_{0}\left(\xi_{0}\right) J_{0}\left(\xi_{d}\right)-\mathrm{j}\left[J_{0}\left(\xi_{0}\right) Y_{1}\left(\xi_{d}\right)-Y_{0}\left(\xi_{0}\right) J_{1}\left(\xi_{d}\right)\right]}{-\mathrm{j}\left[J_{0}\left(\xi_{d}\right) Y_{1}\left(\xi_{0}\right)-Y_{0}\left(\xi_{d}\right) J_{1}\left(\xi_{0}\right)\right]-\left[J_{1}\left(\xi_{d}\right) Y_{1}\left(\xi_{0}\right)-Y_{1}\left(\xi_{d}\right) J_{1}\left(\xi_{0}\right)\right]} .
$$

In order to determine the physical properties of the investigated structure, it is necessary, as the first step, to develop a mathematical model that describes sufficiently physical processes leading from the optical excitation to the thermal response (direct problem), and then to solve the inverse problem of determining the physical properties of the system once the optical excitation, thermal response and model are known. The inverse problem is usually solved by application of curve-fitting. However, non-linear fitting being a rather complex procedure, which quite often gives ambiguous results, it is worthy of effort to investigate approximate solutions of the problem that enable determining of the physical properties of the system without non-linear curve-fitting, by analyzing certain ranges of PT spectra only. Consequently, in this paper amplitude and phase spectra of coatings in the range of high frequencies are presented

$$
\begin{aligned}
& R_{p}=\frac{S_{0} F}{D_{\mathrm{T}} C_{p 0}} \sqrt{\frac{D_{\mathrm{T}}}{\omega}}\left(1+\omega^{2} \tau^{2}\right)^{1 / 4}, \\
& \varphi_{p}=-\frac{\pi}{4}+\frac{\arctan \omega \tau}{2}
\end{aligned}
$$

where

$$
\begin{aligned}
& \mu=\sqrt{\frac{D_{\mathrm{T}}}{\omega \sqrt{1+\omega^{2} \tau^{2}}}}, \quad q=\frac{d}{\mu}, \quad \lambda=1+\alpha d, \\
& \theta=\frac{3 \pi}{4}+\frac{\arctan \omega \tau}{2} .
\end{aligned}
$$

\section{Conclusion}

We have presented an analytical procedure for the calculation of surface temperature in thermally inhomoge- neous coatings induced by an external heat source. The generalized problem of photothermal wave propagation in optical opaque media with constant thermal diffusivity $D_{\mathrm{T}}$ and linearly varying specific heat capacity $C_{p}(x)$ is solved, including thermal memory effect. The case of inhomogeneous surface layers i.e. coatings is discussed. The approximate expressions for FT spectra are given in the range of high frequencies which can make easier FT signal analysis and inverse calculation procedure in FT modulation spectroscopy.

\section{References}

[1] S. Galovic, Ph.D., Elektrotehnicki fakultet, Univerzitet u Beogradu, 2003.

[2] J. Fivez, J. Thoen, J. Appl. Phys. 75, 7696 (1994).

[3] S. Galovic, M.D. Dramicanin, J. Phys. D, Appl. Phys. 32, 1511 (1999).

[4] P. Xiao, Y. Cui, R.E. Imhof, Nondestructive Testing and Evaluation 21, 141 (2006).

[5] F. Macedo, A. Goren, F. Vaz, J.L. Nzodoum Fotsing, J. Gibkes, B.K. Bein, Vacuum 82, 1461 (2008).

[6] J. Cao, J. Phys. D, Appl. Phys. 33, 200 (2000).

[7] H. Hu, X. Wang, X. Xu, J. Appl. Phys. 86, 3953 (1999).

[8] S. Galovic, D. Kostoski, J. Appl. Phys. 93, 3063 (2003).

[9] J. Fivez, J. Thoen, J. Appl. Phys. 75, 7696 (1994).

[10] R.W. Jones, J.F. McClelland, Appl. Spectrosc. 56, 409 (2002).

[11] G.N. Watson, Theory of Bessel Functions, Cambridge: University Press, Cambridge 1944. 